\title{
Facial emotion recognition in schizophrenia: neuropsychological and psychosocial correlates
}

\author{
Jan Jaracz , Marta Grzechowiak, Lucyna Raczkowiak, Janusz Rybakowski \\ From $1^{\text {st }}$ International Congress on Neurobiology and Clinical Psychopharmacology and European \\ Psychiatric Association Conference on Treatment Guidance \\ Thessaloniki, Greece. 19-22 November 2009
}

\section{Background}

There is evidence that facial emotion recognition is disturbed in schizophrenic patient's [1] and is associate with other neurocognitive deficits [2]. Some evidence suggest that affect recognition is an important aspect of psychosocial functioning of patients with schizophrenia [3]. In this study we assessed recognition of facial emotional expression in schizophrenic patients and its relationship with selected clinical and neuropsychological variables as well as with social functioning.

\section{Materials and methods}

Twenty-three patients (mean age 32,3 SD 8,7; mean duration of illness 100 months SD 80) who met the DSM-IV criteria for schizophrenia-paranoid type, hospitalized at the Department of Adult Psychiatry University of Medical Sciences in Poznań, Poland were involved in the study. At time of testing the mean PANSS score was 86,6 (SD 17,7). To assess facial emotion recognition we applied the computerized Penn Emotional Facial Recognition (ER40) task [4]. Cognitive performance was studied using Wisconsin Card Sorting Test. Social functioning was measured with Social Functioning Scale. The control group of healthy volunteers matched for gender and age was included.

\section{Results}

Patients performed worse than control group on the total correct responses, particulary recognition of faces expressing fear $(\mathrm{p}=0.002)$ and sadness $(\mathrm{p}=0.02)$. The median time for correct response was significantly longer $(\mathrm{p}=0.004)$ in schizophrenic group. Patients gave more positive anger, happy and neutral responses, and need more time to identify correctly expression of fear $(\mathrm{p}=0.005)$, happy $(\mathrm{p}=0.03)$ neutral $(0.03)$ and sad $(p=0.005)$ faces. The number of correct responses correlated negatively with number of preservative errors. There was no association of facial emotion expression recognition and social functioning was found.

\section{Conclusions}

Schizophrenic patients performed worse on emotion recognition test than control group. Dysfunction of prefrontal cortex may negatively influence the recognition of emotions.

Published: 22 April 2010

\section{References}

1. Kohler CG, Turner TH, Bilker WB, Brensinger CM, Siegel SJ, Kanes SJ, Gur RE, Gur RC: Facial emotion recognition in schizophrenia: intensity effects and error pattern. Am J Psychiatry 2003, 160:1768-74.

2. Sachs G, Steger-Wuchse D, Kryspin-Exner I, Gur RC, Katschnig H: Facial recognition deficits and cognition in schizophrenia. Schizophr Res 2004, 68:27-35.

3. Hofer A, Benecke C, Edlinger M, Huber R, Kemmler G, Rettenbacher MA, Schleich G, Wolfgang Fleischhacker W: Facial emotion recognition and its relationship to symptomatic, subjective, and functional outcomes in outpatients with chronic schizophrenia. Eur Psychiatry 2009, 24:27-32.

4. Gur RC, Erwin RJ, Gur RE, Zwil AS, Heimberg C, Kraemer HC: Facial emotion discrimination: II. Behavioral findings in depression. Psychiatry Res 1992, 42:241-51.

\section{doi:10.1186/1744-859X-9-S1-S206}

Cite this article as: Jaracz et al:: Facial emotion recognition in

schizophrenia: neuropsychological and psychosocial correlates. Annals of General Psychiatry 2010 9(Suppl 1):S206. 